\title{
New Theoretical and Observational Results on Transverse Magnetic Fluctuations near the Magnetopause
}

\author{
G. Gnavi ${ }^{1}$, C. J. Farrugia ${ }^{2}$, and F. T. Gratton ${ }^{1}$ \\ ${ }^{1}$ Instituto de Física del Plasma, CONICET and FCEyN-Universidad de Buenos Aires, Buenos Aires, Argentina \\ ${ }^{2}$ Institute for the Study of Earth, Oceans, and Space, Space Research Center, \\ and Department of Physics, University of New Hampshire, Durham, NH, USA
}

Received on 6 February, 2004

\begin{abstract}
Electromagnetic ion cyclotron waves in the plasma depletion layer measured by Wind on three inbound passes of the magnetosheath near the stagnation streamline are modeled using theoretical results from Gnavi et al., $J$. Geophys. Res., 105, 20973, 2000. The kinetic dispersion relation in a plasma composed of electrons, protons, and $\alpha$ particles, is solved with each species modeled by a bi-Maxwellian distribution function with parameters taken from observations, where available, and from average values found in the literature. While one pass was under substantially high solar wind dynamic pressure $(\sim 6.4 \mathrm{nPa})$, the other two passes were under normal dynamic pressure at $1 \mathrm{AU}(\sim 2.2 \mathrm{nPa})$. The presence of electromagnetic ion cyclotron waves in the terrestrial plasma depletion layer under normal dynamic pressure is documented and analyzed for the first time. The power spectral density of the magnetic fluctuations transverse to the background field, using high resolution $(\sim$ 11 samples/s) data from the Magnetic Field Investigation, is obtained for the inner, middle and outer regions of the plasma depletion layer. The analysis of spectra and comparison with theory is extended to the normal dynamic pressure regime. The observations show that at the inner plasma depletion layer position the spectral power density weakens as the dynamic pressure decreases, and that the frequency range of emission shifts downward with diminishing pressure. Using bipolytropic laws for the anisotropic magnetosheath, we argue that the effect of a reduction of $P_{d y n}$ is to lower $A_{p}$, thereby weakening the driver of EICWs leading to marginally bifurcated spectra and weaker EICW activity in the PDL under typical conditions. Qualitative and in some cases quantitative agreement between theory and data is very good.
\end{abstract}

\section{Introduction}

The theory of the flow of the magnetosheath around the magnetosphere developed by Spreiter and coworkers in the sixties has been the cornerstone of the subject and very successful. It gave origin to the Convected Field Gas Dynamic (CFGD) model, which is the weak magnetic field limit of magnetohydrodynamics. This simplification gives good results for the stand-off distance of the bow shock and the general geometry of the field in the magnetosheath. Near the magnetopause the field starts to increase and to pile up against the magnetopause, particularly when the field is not removed by reconnection. This pile-up vitiates the basic assumption of the CFGD model. This feature gives a possible characterization of the plasma depletion layer (PDL) as a thin layer dependent on the Alfvén Mach number where the magnetic field increases and simultaneously the density decreases [1].

The PDL can also be characterized by its wave activity. Anderson, Fuselier and coworkers [2] have shown that the PDL has a peculiar wave activity: the electromagnetic ion cyclotron waves (EICWs). These are transverse waves, contrasting strongly with the compressional mirror modes ubiquitous in the main body of the magnetosheath outside the PDL. Until now their study has been carried out under compressed magnetosphere conditions, rather an unusual situation, based on data recorded by AMPTE/CCE. This space- craft sampled the magnetosheath when the solar wind dynamic pressure was $P_{d y n} \geq 5.2 \mathrm{nPa}$. The more typical condition of lower $P_{d y n}$ was not investigated. The first theoretical study was developed by Gnavi, Gratton, and Farrugia [3] using a statistical data analysis of Phan et al. [4], characteristic of normal pressures.

In this paper we explore EICWs in the PDL for normal dynamic pressure values not previously considered. The theory of Gnavi, Gratton, and Farrugia is confronted with data from three passes across the magnetopause by Wind in 1994. These passes are most appropriate for the study of PDL wave activity since they were very close to the stagnation streamline, where the influence of the field on the flow (and hence the strength of depletion) is most pronounced.

The change of EICW spectral activity from high to normal dynamic pressure is examined and an explanation presented for several observed features, using the theory of gyromagnetic emission in a multi-component plasma. In the discussion section, an argument showing how a reduction of solar wind $P_{d y n}$ leads to a significant weakening of EICW activity is presented.

\section{Theory}

In the present model the plasma is composed of protons, alpha particles and electrons having bi-Maxwellian distribu- 
tion functions that are expressed as

$$
f_{\text {Max }}^{0}=\left(\frac{1}{\pi}\right)^{3 / 2} \frac{n}{w_{\|} w_{\perp}^{2}} \exp \left(-\frac{v_{\|}^{2}}{w_{\|}^{2}}\right) \exp \left(-\frac{v_{\perp}^{2}}{2 w_{\perp}^{2}}\right) .
$$

$T_{\|}$and $T_{\perp}$ denote the parallel and perpendicular temperatures, respectively. The spatio-temporal dependence of the waves is $\exp (i[k z-\omega t])$, with the $z$ axis aligned along the background magnetic field $B_{0} ; k$ is the wave number, and $\omega=\omega_{r}+i \gamma$ the complex frequency. Quantity $\omega_{r}=\operatorname{Re}(\omega)$ is the angular frequency of the wave, and $\gamma=\operatorname{Im}(\omega)$ is the growth $(\gamma>0)$ or damping $(\gamma<0)$ rate. The species will be indexed by $s$ ( $s=p, \alpha, e$, for protons, alphas, and electrons, respectively). The relative density of the $\alpha$ s to the protons will be denoted by $\eta_{\alpha}$. For each component $s$ the quantities $w_{\|, s}=\sqrt{2 k_{B} T_{\|, s} / m_{s}}$ and $w_{\perp, s}^{2}=\sqrt{2 k_{B} T_{\perp, s} / m_{s}}$ are the thermal speeds parallel and perpendicular to the magnetic field, respectively.

The linear dispersion relation, according to the Vlasov self-consistent field model, for left hand polarized EICWs, propagating along the magnetic field lines $(\vec{k} \| \vec{B})$, is given by

$$
k^{2} c^{2}=\omega^{2}+\sum_{s} \omega_{p, s}^{2}\left[A_{s}+\frac{\left(A_{s}+1\right) \omega-A_{s} \Omega_{s}}{k w_{\|, s}}\right] Z_{0, s}\left(\frac{\omega-\Omega_{s}}{k w_{\|, s}}\right),
$$

where $k$ stands for $k_{\|}$, for simplicity, and the sum extends over the species. The thermal anisotropy is $A_{s}=$ $\left[\left(T_{\perp} / T_{\|}\right)_{s}-1\right], \omega_{p, s}$ is the plasma frequency and $\Omega_{s}$ the gyrofrequency of species $s$. Finally, $Z_{0}$ is the modified plasma Zeta function [5].

An ion (proton or alpha particle) moving with a particular velocity component $v_{\|}^{s}(s=p, \alpha)$ along the field line, can only emit or absorb EICWs when the following resonance condition is satisfied [6]

$$
\omega_{r}(k)-k v_{\|}^{s}=\Omega_{s} \quad(s=p, \alpha),
$$

so that the particle gyrates in concert with the transverse electric field of the wave at the Doppler-shifted frequency observed in the moving frame of the ion.

For bi-Maxwellian distribution functions, the condition for wave growth, i.e., that there be more emitters than absorbers of a given ion species at any given frequency $\omega_{r}(k)$ can be written as

$$
\overline{\frac{d f_{0, s}}{d v_{\|}}}\left(v_{\|}^{*}\right)>0
$$

where

$$
\frac{\overline{d f_{0, s}}}{d v_{\|}}\left(v_{\|}^{*}\right) \propto\left[\Omega_{s}\left(\frac{1}{w_{\|, s}^{2}}-\frac{1}{w_{\perp, s}^{2}}\right)-\frac{\omega_{r}(k)}{w_{\|, s}^{2}}\right] \overline{f_{0, s}}\left(v_{\|}^{*}\right) .
$$

Here, the function $\overline{f_{0, s}}\left(v_{\|}\right)$is the bi-Maxwellian averaged over the perpendicular velocity $v_{\perp}$, and $v_{\|}^{*}$ is the peculiar $v_{\|}^{s}$ value that satisfies equation $3, v_{\|}^{*}=\left(\omega_{r}(k)-\Omega_{s}\right) / k$, for each species. When inequality 4 is reversed, the wave is damped instead.

As can be seen from 5, the number of emitters (or absorbers) at a given frequency of the wave, is proportional to

$$
\exp \left[-\left(\left(\omega_{r}(k)-\Omega_{s}\right)^{2} / k^{2} w_{\|, s}^{2}\right)\right],
$$

i.e., to the number of resonant ions. Equation 5 gives a frequency limit, $\omega_{s}^{l}$, for each species, that separates the range of $\omega_{r}(k)$ in which the ions are predominantly emitters, $\omega_{r}<\omega_{s}^{l}$, from the range $\omega_{r}>\omega_{s}^{l}$, where the particles of that species absorb energy from the wave instead. The frequency limit for each species is given by

$$
\omega_{s}^{l}=\frac{A_{s}}{A_{s}+1} \Omega_{s}
$$

Since in our case $A_{s}>0$ the frequency limit $\omega_{s}^{l}$ is smaller than the gyrofrequency of each ion component.

In the low frequency band both $p$ and $\alpha$ contribute to the growth of the waves, with a negligibly small growth rate at very low frequencies in the Alfvén wave regime. The rate of growth increases for higher frequencies until the $\omega_{\alpha}^{l}$ limit is reached. The protons are emitters until they reach their limit frequency $\omega_{p}^{l}$. Above that value there is absorption only.

\section{PDL data measured by Wind near the stagnation streamline}

The data available for the present analysis are magnetic field and proton observations made by Wind on three inbound low-latitude magnetosheath passes on December 24, November 30, and December 12, 1994, within one hour of local noon. These are: the GSM $B_{x}, B_{y}, B_{z}$ components of the magnetic field, the total field, the proton density, temperature, and bulk speed, the temperature ratio $T_{p, \perp} / T_{p, \|}$, and the proton plasma betas parallel and perpendicular to the magnetic field. Electron parameters have only a minor effect on EICW excitation [3] and are available only on the December 24, 1994 pass. They indicate that in the magnetosheath the electron temperature is approximately ten times lower than the proton temperature. Also, the electron temperature ratio $T_{e, \perp} / T_{e, \|} \sim 1$.1. Similar characteristics will be assumed for those ratios in all three passes. The magnetic field and plasma observations were made by the Magnetic Field Investigation [7] and the 3D Plasma Analyzer [8] on Wind, and are given at a resolution of $3 \mathrm{~s}$ and $51 \mathrm{~s}$, respectively. For the analysis of magnetic field fluctuations in the PDL the 0.09 s resolution magnetic field data are used. 
TABLE 1. Parameters for the computation of theoretical growth and damping rates.

\begin{tabular}{cccccccc} 
Date & Region & Interval (UT) & $A_{p}$ & $\beta_{p, \|}$ & $f_{p}$ & $L 1$ & $L 2$ \\
Nov. 30, 1994 & Inner & $21: 20-21: 23$ & 1.25 & 0.30 & 0.77 & 0.44 & 0.25 \\
& Middle & $21: 13-21: 16$ & 1.04 & 0.69 & 0.62 & 0.32 & 0.19 \\
& Outer & $21: 05-21: 08$ & 0.66 & 1.13 & 0.56 & 0.22 & 0.15 \\
Dec. 12, 1994 & Inner & $13: 52-13: 55$ & 0.98 & 0.66 & 0.68 & 0.33 & 0.21 \\
& Outer & $13: 46-13: 49$ & 0.80 & 0.83 & 0.66 & 0.29 & 0.19 \\
Dec. 24, 1994 & Inner & $11: 22-11: 27$ & 1.61 & 0.24 & 1.44 & 0.89 & 0.50 \\
& Middle & $11: 16-11: 21$ & 1.05 & 0.51 & 1.29 & 0.66 & 0.40 \\
& Outer & $11: 06-11: 11$ & 0.81 & 0.84 & 1.14 & 0.51 & 0.33 \\
\hline
\end{tabular}

\section{Spectral Analysis}

In the present analysis, the PDL is divided into regions. The one that is closest to the magnetopause will be called inner and the region that is farthest from it outer. In two scenarios, there is a third region, called middle, located between the inner and outer ones. In Table 1 the proton parameters for the different regions are given. $A_{p}$ is highest in the inner region and lowest in the outer one. The opposite situation holds for $\beta_{\|}$.

The power spectral density (PSD) for the intervals is shown in Figs. 1-3. Plotted are the left-hand (heavy solid line), right hand (dot-dashed line) and parallel (dashed trace) PSDs. The vertical dot-dash lines in each panel give the proton and $\alpha$-particle gyrofrequencies. The vertical lines labeled $L 1$ and $L 2(L 1>L 2)$ refer to the theoretical limiting frequencies discussed in section 2 . Thus, below frequency $L 2, \alpha$ s give up energy to the wave and so do protons below $L 1$. Above the respective $L$-line alpha particles and protons absorb energy from the wave. Between $L 2$ and $L 1$ there is competition between alpha absorption and proton emission. The direction of energy exchange depends on the alpha concentration and on the value of the respective $\beta \mathrm{s}$. Figs. 1-3 show that frequencies $L 1$ and $L 2$ shift downwards from inner to outer PDL regions.

\subsection{December 24, 1994}

In this pass the PDL is divided into three regions: outer (11:06 - 11:11 UT), middle (11:16 - 11:21 UT), and inner (11:22 - 11:27 UT) as presented in Figure 1.

There are two peaks in the outer region. One at $0.25 \mathrm{~Hz}$ $(<L 2)$ and a weaker peak at $0.42 \mathrm{~Hz}(<L 1)$.

The main activity in the middle region is from $0.2 \mathrm{~Hz}$ to $0.4 \mathrm{~Hz}(<L 2)$, with a minor proton contribution just below the $L 1$ frequency.

For the inner region, emission is due mainly to alphas and is strong from $0.25 \mathrm{~Hz}$ to $0.5 \mathrm{~Hz}(\leq L 2)$. Above the alpha gyrofrequency and below $L 1$ there is a weaker emission band from the protons. Above $L 1$ there is some weak activity.

Temperature anisotropy values on December 24, 1994, are the highest of the three passes.

\subsection{November 30, 1994}

The PDL is divided for this pass into outer (21:05 - 21:08 UT), middle (21:13 - 21:16 UT), and inner (21:20 - 21:23 UT).

Figure 2 shows the power spectral density in the three regions of the PDL on November 30, 1994, in the same format of Figure 1. With respect to December 24, there is weaker EICW activity on November 30, and it takes place at lower frequencies.

For the outer region the power lies in frequencies lower than the alpha gyrofrequency. For higher frequencies there is a sharp drop in power.

In the middle region power is also mainly on the lower frequencies. The power spectral density between $L 1$ and $L 2$ is lower than in the outer region due to the prevailing lower $\beta_{p, \|}$ (see Table 1).

In the inner region there is one local power peak below the alpha resonance at $0.25 \mathrm{~Hz}$ near $L 2$, and a weaker one at $0.43 \mathrm{~Hz}$ near $L 1$. The first one is mainly due to the alphas and the one at higher frequencies to proton emission.

From the outer region to the inner, between $L 2$ and $L 1$ the PSD decreased in accordance with the change in $\beta_{p, \|}$ from 1.13 to 0.3 .

\subsection{December 12, 1994}

For this pass only two regions have been selected due to a marked time variability of the data. The outer region is in the interval 13:46 - 13:49 UT and the inner one from 13:52 to $13: 55$ (see Figure 3).

For the outer region the power resides well below the alpha gyrofrequency, in the range 0.1 to $0.25 \mathrm{~Hz}$. There is a continuous decrease in power.

In the inner PDL the power is mainly at frequencies less than $0.21 \mathrm{~Hz}(=L 2)$. There is a weaker band centered around $L 1$ (this value is very close to the alpha gyrofrequency). 


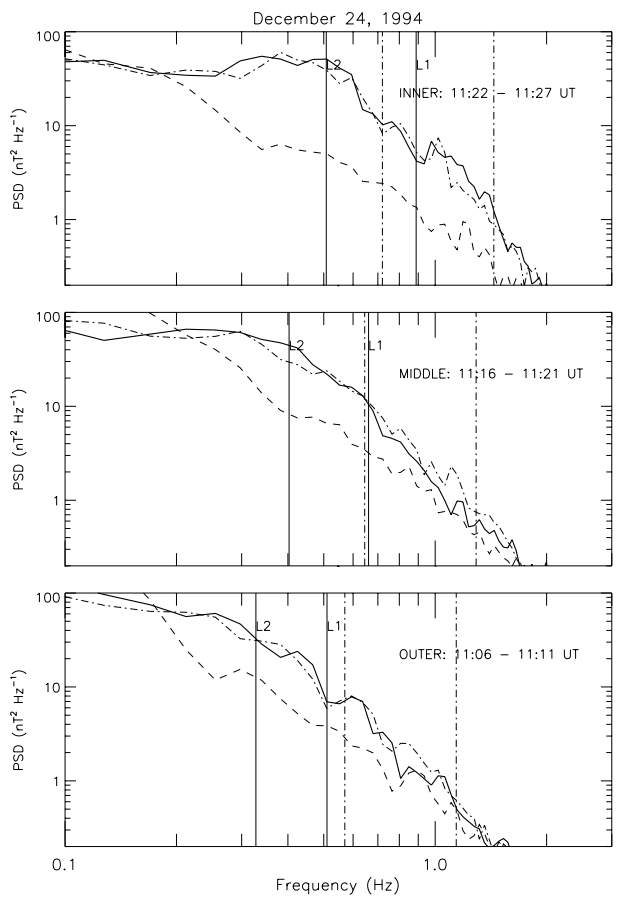

Figure 1. Power spectral density $\left(\mathrm{nT}^{2} / \mathrm{Hz}\right) v s$. frequency $(\mathrm{Hz})$ in the inner, middle and outer regions of the PDL (from top to bottom) on December 24, 1994. Left-hand (heavy solid line), right-hand (dot-dashed line) and parallel (dashed trace) PSDs. The vertical dot-dash lines are drawn at the proton and $\alpha$-particle gyrofrequencies. The vertical lines $L 1$ and $L 2$ mark the theoretical limiting frequencies discussed in the text.

\section{Growth and damping rates: theory and observations}

It is important to note that there is no straightforward relation between the theoretical values describing the linear stage of the instability and the observed values that could be related to saturation effects in a nonlinear regime. Nevertheless, the results from the linear theory should be indicative of the ranges where growth or damping takes place. Linear mechanisms (such as alpha-proton differential drifts or high $A_{\alpha}$ with low $\beta_{p, \|}$ effects.) that close gaps in emission, thus producing continuous spectra, have been discussed in Ref. 3. Also, nonlinear wave interaction could produce cascades towards lower frequencies and new waves in ranges forbidden by the linear theory. Therefore, full agreement between theory and observations may not occur.

Figures 4-6 show the results of the numerical solutions of the dispersion relation in a log-log representation. Growth (solid lines) and damping rates (dashed lines), normalized to the proton gyrofrequency $\left(\Omega_{p}\right)$, are plotted versus frequency, $f$, in Hz. The low frequency range of EICWs is the undamped Alfvén wave regime.

The numerical values used in the calculations are those in Table 1. Quantities $A_{p}$ and $\beta_{p, \|}$ are averages over the subdivisions of the PDL taken from Wind measurements. Quantity $\eta_{\alpha}$ is assumed to be 0.04 , a typical solar wind va- lue. The values of $A_{\alpha}$ and $\beta_{\alpha, \|}$ are taken from the literature. We set $T_{\alpha} / T_{p}=4$ ([9] and references therein). For $A_{\alpha}$, we assume $T_{\alpha, \perp} / T_{\alpha, \|}=1.25 T_{p, \perp} / T_{p, \|}$, which agrees approximately with values measured by AMPTE/CCE [10]. Electrons are assumed to be isotropic and $T_{p} / T_{e}=10$.
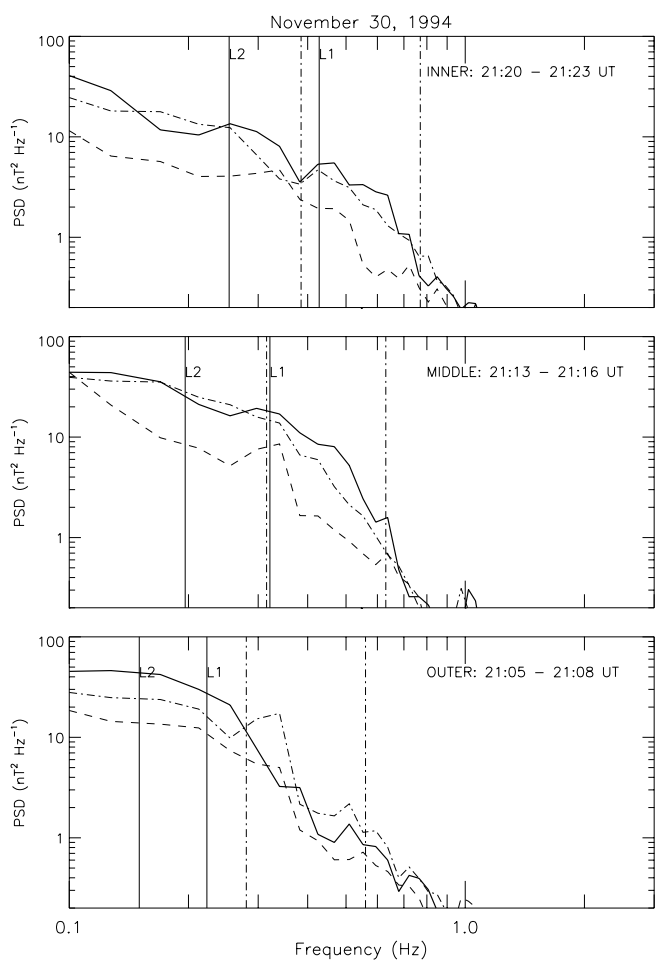

Figure 2. Similar to Figure 1 but for November 30, 1994.
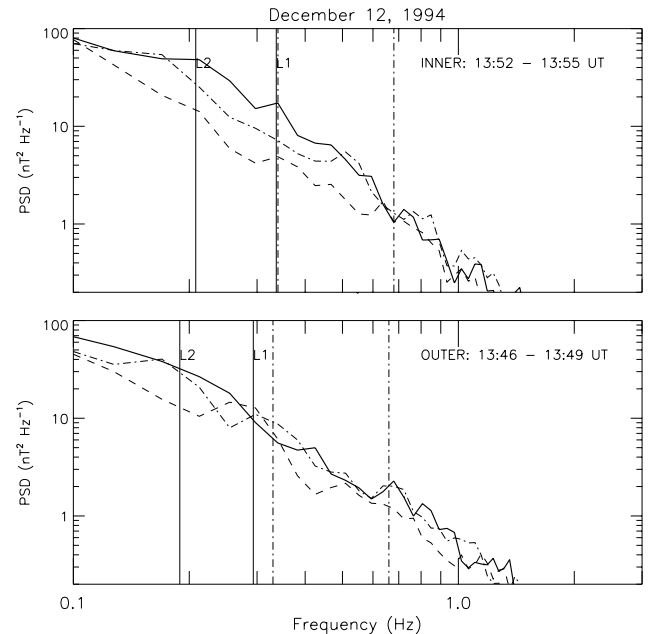

Figure 3. Power spectral density $\left(\mathrm{nT}^{2} / \mathrm{Hz}\right) v s$. frequency $(\mathrm{Hz})$ in the inner and outer regions of the PDL on December 12, 1994.

In all passes, both theory and data show a shift of the active band to lower frequencies as the PDL is crossed from inner to outer region. 
The forthcoming detailed comments will show that there is a reasonable agreement between the linear theory of EICWs emission and measured power spectral density for both high (December 24) as well as normal (November 30 and December 12) dynamic pressure.
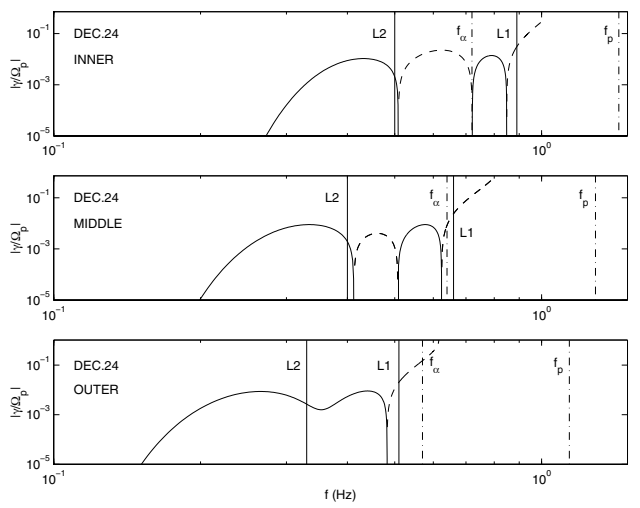

Figure 4. Growth rates vs. frequency $(\mathrm{Hz})$ predicted by the linear theory for the three regions of the PDL on December 24, 1994. Solid curves denote growth and dashed curves represent damping rates.
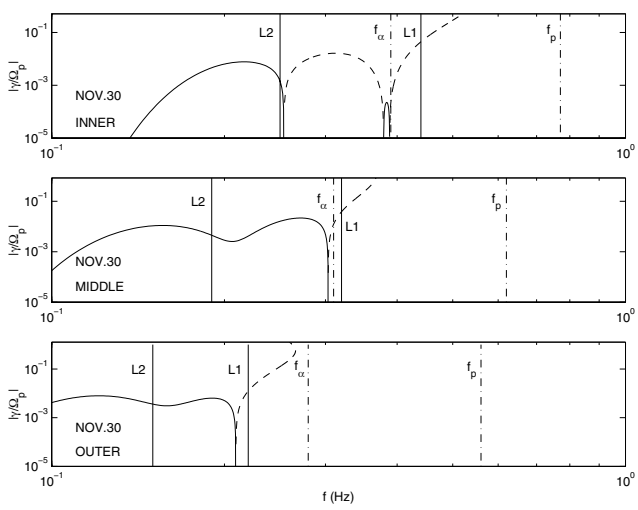

Figure 5. Similar to Figure 4 but for November 30, 1994.

\subsection{December 24, 1994}

Inner PDL: Figure 4 presents the theoretical results. Two activity ranges are present, separated by a band of damped frequencies. The alpha emission extends from $\sim 0.35$ to $0.5 \mathrm{~Hz}$, with a peak at $0.42 \mathrm{~Hz}$. There is good agreement with the observed results in the top panel of Figure 1. The theoretical peak due to proton emission at $0.80 \mathrm{~Hz}$ is also observed in Figure 1 just below $L 1$. The bifurcated spectrum presented by the theory is weakly reflected in the data. Above $\sim 0.85 \mathrm{~Hz}$ the damping is very strong.
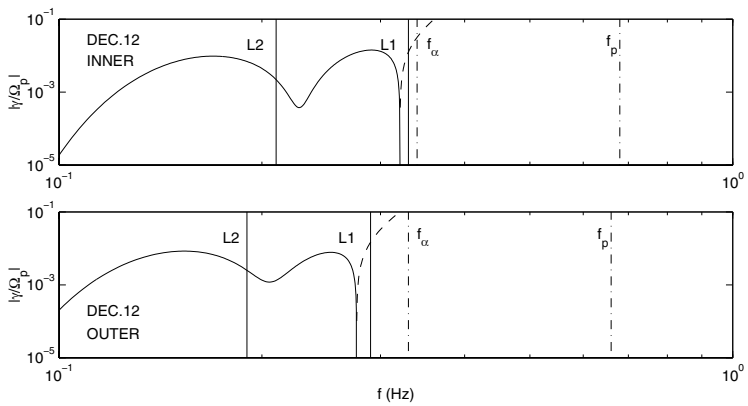

Figure 6. Similar to Figure 4 but for the inner and outer regions of the PDL on December 12, 1994.

Middle PDL: According to theory there is a broad alpha peak, centered at $0.34 \mathrm{~Hz}$, and a narrow proton peak at 0.58 Hz. In Figure 1, middle panel, there is a local enhancement at those frequencies. The gap predicted by theory is reflected in the observed PSD as a change in the slope of the spectrum just above the $L 2$ frequency. Above $0.62 \mathrm{~Hz}$, the theoretical damping is very large and the measured PSDs are very small.

Outer PDL: A continuous band of activity extending from 0.16 to $0.48 \mathrm{~Hz}$, with lower growth rates around 0.34 $\mathrm{Hz}$ is predicted. The corresponding features in the observations are the two peaks at $0.26 \mathrm{~Hz}$ and $0.20 \mathrm{~Hz}$ (Figure 1, bottom panel). Measured PSDs are very small above $0.5 \mathrm{~Hz}$ with the exception of a weak peak above $L 1$ not predicted by theory.

\subsection{November 30, 1994}

Inner PDL: The $\alpha$ range is wide with a maximum at $0.24 \mathrm{~Hz}$, while the proton range is very narrow and located at 0.38 $\mathrm{Hz}$ (see Figure 5). In the observations the PSD shows local enhancements near these frequencies (Figure 2, top panel). The theoretical value of the frequency of the proton peak is $0.05 \mathrm{~Hz}$ lower than the observed one. In the range between 0.26 and $0.37 \mathrm{~Hz}$ where theory predicts damping, the observed PSD also declines.

Middle PDL: In the middle PDL, a continuous active band stretches from 0.1 to $0.3 \mathrm{~Hz}$. Above this range the damping rate increases very rapidly Two maxima are located at $0.15 \mathrm{~Hz}$ and $0.27 \mathrm{~Hz}$, correponding to alpha and proton emissions, respectively. The maxima are at lower frequencies with respect to the inner PDL case. A similar trend, appears in the observations (see Figure 2, middle panel), where maxima are located at 0.15 and $0.22 \mathrm{~Hz}$.

Outer PDL: The theoretical results for the outer PDL represented in Figure 5 show a continuous band in the range from 0.1 to $0.22 \mathrm{~Hz}$, below the $\alpha$ gyrofrequency, $f_{\alpha}$. For higher frequencies the damping is extremely high. A similar situation appears in the power spectral density (Figure 2, bottom panel) where the observations show a sharp power decrease at $0.25 \mathrm{~Hz}$.

There is very good agreement between theory and observations for the low pressure pass on November 30. For all three PDL regions, activity is shifted towards frequencies lower than those of the high $P_{d y n}$ pass on December 24 . 


\subsection{December 12, 1994}

Inner and Outer PDL: As seen in Figure 6 the theoretical growths for inner and outer regions are similar and both show continuous activity at low frequencies. In the inner region, the emission reaches up to $0.32 \mathrm{~Hz}$ (slightly above $f_{\alpha}$ ). In the outer PDL the active region ends at $0.28 \mathrm{~Hz}$, far from the alpha resonance, exactly as in the data. Activity at higher frequencies is heavily damped according to theory.

\section{Discussion}

\subsection{Effect of $P_{d y n}$ on EICW activity in the PDL}

The observed values of the temperature anisotropy $A_{p}$ on the Wind passes given in Table 1 are significantly lower than those of the AMPTE/CCE measurements and the correspon$\operatorname{ding} \beta_{p, \|}$ are higher (compare our Table 1 with Table 2 of Ref. 11). A semi-quantitative argument is presented to show that near the stagnation streamline, the lower $A_{p}$ is due to the lower $P_{d y n}$.

Studying the anisotropic magnetosheath, Hau et al. [12] and Farrugia et al. [13] have empirically established that near the subsolar region the MHD equations can be closed by a double-polytropic law of the form

$$
\frac{p_{\perp}}{\rho}=c_{\perp}, \frac{p_{\|} / B^{1 / 2}}{\rho^{3 / 2}}=c_{\|},
$$

where $c_{\perp}, c_{\|}$, are constants. These equations do not conserve the magnetic moment of the ions as an adiabatic invariant, because of the high frequency magnetic fluctuations in the magnetosheath. It follows that

$$
\frac{T_{\perp}}{T_{\|}}=\frac{p_{\perp}}{p_{\|}}=\frac{c_{\perp}}{c_{\|}} \sqrt{\frac{B}{\rho}} .
$$

On the other hand, a well known consequence of the MHD frozen-in field condition, stated in a Lagrangian description, can be written as

$$
\frac{\mathbf{B}}{\rho}=\left(\frac{\mathbf{B}}{\rho}\right)_{0} \cdot \operatorname{grad}(\mathbf{s}),
$$

where $\mathbf{s}$ is the Lagrangian displacement (see, e.g., Ref.14). The last equation leads to

$$
\frac{\left(\frac{B}{\rho}\right)_{2}}{\left(\frac{B}{\rho}\right)_{1}}=\frac{\delta l_{2}}{\delta l_{1}},
$$

which describes the intensification of the magnetic field by the stretching of magnetic field lines from position 1 to position 2 . Here $\delta l_{1}$ is the distance between two plasma elements on a given magnetic field line, which is mapped into $\delta l_{2}$ as the plasma flow is diverted around the magnetosphere; $B_{1,2}$ denote the magnetic field strength at positions 1 and 2, respectively [14].

Any given segment of a magnetic field line, mapped by the plasma motion near the stagnation streamline, is stretched more for high than for low $P_{d y n}$ values. In fact, the ra- dius of curvature of the frontside magnetopause at the subsolar point is larger when $P_{d y n}$ is smaller. In the schematic of Figure 7 the magnetopause is sketched for two different values, $\left(P_{d y n}\right)_{s},\left(P_{d y n}\right)_{h}$, where $s$ stands for small (the left-hand sketch) and $h$ for high pressure (the right-hand sketch). The magnetopause appears as a blunter obstacle to the flow when the pressure is low. Two plasma elements are shown on the near stagnation streamlines, separated by a distance $\delta l_{1}$. On being diverted past the magnetopause, the distance between the plasma elements $\delta l_{2}$ is larger for the higher pressure, i.e., $\delta l_{2, h}>\delta l_{2, s}$, because the speed of the diverted plasma is higher. This is in agreement with the known fact that the magnetic field pile-up close to the magnetopause becomes stronger for compressed conditions (compare, for example, the magnetic field strength at the subsolar magnetopause on December 24, when the pressure is high, with that on November 30, when the pressure is low, Figs. 1 and 2).

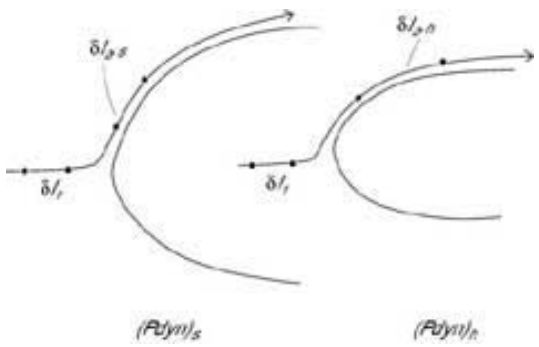

Figure 7. A schematic showing a compressed magnetopause on the right and a dilated one on the left. Two plasma elements at a distance $\delta l_{1}$ are mapped to two different distances $\delta l_{2 s}$ and $\delta l_{2 h}$ along the off-solar magnetopause, the one for the compressed magnetopause being longer.

Applying the empirical relation, Eq.8, we finally obtain

$$
\frac{\left(\frac{T_{\perp}}{T_{\|}}\right)_{h}}{\left(\frac{T_{\perp}}{T_{\|}}\right)_{s}}=\left(\frac{\delta l_{2, h}}{\delta l_{2, s}}\right)^{1 / 2} .
$$

Hence,

$$
\frac{\left(\frac{T_{\perp}}{T_{\|}}\right)_{h}}{\left(\frac{T_{\perp}}{T_{\|}}\right)_{s}}>1,
$$

when

$$
\left(P_{d y n}\right)_{h}>\left(P_{d y n}\right)_{s} .
$$

The temperature ratio (and hence the thermal anisotropy) thus decreases with decreasing $P_{d y n}$. At lower values of $P_{d y n}$ the EICW activity is expected to be weaker than at higher values, for at least two reasons. (1) By producing lower $A_{p}$ values, a smaller $P_{d y n}$ generates conditions of smaller growth rates by weakening their driver (i.e., the free energy available). Further, excitation is pushed to lower frequencies, because frequencies $L 1$ and $L 2$ (measured in units of $f_{p}$ ) are shifted downwards. (2) Close to the (low-shear) magnetopause, $P_{d y n} \propto B^{2}$ (by pressure balance). Therefore, a smaller $P_{d y n}$ implies a smaller $f_{p}(=q B / 2 \pi m c)$, which brings an additional diminution of the absolute values of the growth rates $\left(\propto f_{p}\right)$ and the further shift of the EICW activity to lower frequencies). 


\subsection{Classification of magnetic spectral types}

AMPTE/CCE studies referred to compressed magnetospheric conditions with a solar wind $P_{d y n}$ equal to, or larger than, the one of the December 24, 1994 example. On the other hand, November 30, and December 12, 1994, passes were under typical $P_{d y n}$ at $1 \mathrm{AU}(\sim 2.2 \mathrm{nPa})$. Therefore, relevant differences must appear in a comparison of the observational results of the present study with those of Refs. 11 and 15. These authors classified the magnetic spectral types in the magnetosheath. Inside the PDL they identified bifurcated (BIF), continuous (CON), and low (LOW) spectra. The average values of the parameters $A_{p}$ and $\beta_{p, \|}$ characterizing the categories (see Table 2 in Ref. 11) are: $A_{p}=2.14,0.96$, and $0.83 ; \beta_{p, \|}=0.22,1.11$, and 1.25 for BIF, CON, and LOW respectively. This shows a systematic decrease in $A_{p}$ and a corresponding increase in $\beta_{p, \|}$ from BIF to LOW, which according to Anderson et al. [11] implies a spatial classification. Similar criteria, applied to the spectra discussed in this study give: December 24 (INNER) and November 30 (INNER) are (marginally) BIF spectra; December 24 (MIDDLE and OUTER) and November 30 (MIDDLE) are CON spectra; November 30 OUTER and December 12 (INNER and OUTER) are LOW spectra.

As a consequence of the results in section 6.1 and the comparison with Ref. 11, it is reasonable to predict that under normal solar wind pressure there will be a preponderance of spectra of the CON and LOW types in the PDL, and few BIFs, if any. Also EICW activity in the PDL weaker than under compressed conditions is expected.

\section{Conclusions}

The presence of EICWs in the terrestrial PDL under normal $(\sim 2.2 \mathrm{nPa})$ solar wind dynamic pressure has been documented and analyzed for the first time. The analysis of EICW spectra and theory has been extended to this more typical $P_{d y n}$ regime.

The agreement of the power spectral densities with theoretical predictions was qualitatively, and at times even quantitatively, very good. Theory and observations agreed on frequency where the activity peaks, the width of active bands, and the limiting frequencies $L 1$ and $L 2$. The discrepancies encountered in some cases may have diverse origins. Nonlinear effects such as parametric decays and turbulent cascading to lower frequencies were not studied. Also, the assumption of spatial uniformity of the plasma properties inherent of the infinite plasma model is not realized in practice.

The dependence of properties of EICW emission on the front side of the magnetopause on the dynamic pressure of the solar wind is analyzed. Thus, a microscopic process - ion gyromagnetic radiation - is seen to be modulated by a global quantity like $P_{d y n}$. Also, a semi-empirical explanation for the direct correlation between $P_{d y n}$ and $A_{p}$, the thermal anisotropy, in the stagnation point flow region of the PDL is presented.

Gnavi, Gratton, and Farrugia [3] were the first to propose a subdivision of the PDL that exhibits evolutionary trends of the EICW excitation. Under conditions of normal $P_{d y n}(\sim 2$ $\mathrm{nPa}$ ) the power spectral density (PSD) of the waves is mainly of the CON and LOW type. In compressed magnetospheres
( $P_{\text {dyn }}$ much larger than normal) instead, BIF type spectra prevail in the inner part of the plasma depletion layer.

The subdivisions introduced in the analysis reflect bona fide structural categories of the PDL in space, each one defined by a corresponding type of dominant wave activity, which persist in the range of solar wind pressures explored.

It is predicted that under normal solar wind pressure there will be a preponderance of spectra of the CON and LOW types in the PDL, and few BIFs, if any. Also, weaker EICW activity in the PDL than under compressed conditions is expected.

\section{Acknowledgments}

We are grateful to the following investigators that contributed with spacecraft data and made this research possible: H. Matsui and R. Torbert, Space Research Center, UNH; R. P. Lepping, NASA, Goddard Space Flight Center; M. Oieroset and R. P. Lin, Space Science Laboratories, Berkley, CA. Part of this work was done while G.G. and F.T.G. were on a research visit to the Space Research Center of the University of New Hampshire. This work is partially supported by NASA grants NAG5-13116 and NAG5-11803, and Argentinean grants UBACYT X059, UBACYT X291 and CONICET PIP-2013.

\section{References}

[1] B. J. Zwan, and R. A. Wolf, Depletion of solar wind plasma near a planetary boundary, J. Geophys. Res. 81, 1636 (1976).

[2] B. J. Anderson, S. A. Fuselier, and D. Murr, Geophys. Res. Lett. 18, 1955 (1991).

[3] G. Gnavi, F. T. Gratton, and C. J. Farrugia, J. Geophys. Res. 105, 20, 973 (2000).

[4] T. -D. Phan, G. Paschmann, W. Baumjohann, N. Sckopke, and H. Luehr, J. Geophys. Res. 99, 121 (1994).

[5] H. T. Stix, Waves in Plasmas, Am. Inst. Physics, N.Y., 1992.

[6] D. B.Melrose, Instabilities in space and laboratory plasmas, Cambridge University Press, Cambridge, 1986.

[7] R. P. Lepping et al., Space Sci. Rev. 71, 207, 1995.

[8] R. P. Lin et al., Space Sci. Rev. 71, 125 (1995).

[9] B. J. Anderson, S. A. Fuselier, and D. Murr, Geophys. Res. Lett. 18, 1955 (1991).

[10] S. A. Fuselier, D. M. Klumpar, E. G. Shelley, B. J. Anderson, and A. Coates, J. Geophys. Res. 96, 21,095 (1991).

[11] B. J. Anderson, S. A. Fuselier, S. P. Gary, and R. E. Denton, J. Geophys. Res. 99, 5877 (1994).

[12] L. N. Hau, T.-D. Phan, B. U. Ö. Sonnerup, and G. Pashmann, Geophys. Res. Lett. 20, 2255 (1993).

[13] C. J. Farrugia, N. V. Erkaev, D. F. Vogl, H. K. Biernat, M. Oieroset, R. P. Lin, and R. P. Lepping, J. Geophys. Res. 106, 29, 373 (2001).

[14] S. Chandrasekhar, Hydrodynamic and Hydromagnetic stability, Oxford University Press, 1961.

[15] B. J. Anderson, and S. A. Fuselier, J. Geophys. Res. 98, 1461 (1993). 